\title{
Could kDNA-PCR in Peripheral Blood Replace the Examination of Bone Marrow for the Diagnosis of Visceral Leishmaniasis?
}

\author{
Natalia Souza de Godoy, ${ }^{1}$ Marcos Luiz Alves Andrino, ${ }^{1}$ \\ Regina Maia de Souza, ${ }^{1}$ Erika Gakiya, ${ }^{1}$ Valdir Sabbaga Amato, ${ }^{1,2}$ \\ José Ângelo Lauletta Lindoso, ${ }^{3,4}$ and Lucia Maria Almeida Braz \\ ${ }^{1}$ Laboratório de Investigação Médica, Parasitologia LIM 46 do Hospital das Clínicas da Faculdade de Medicina da Universidade de \\ São Paulo (HCFMUSP), Avenida Dr. Enéas Carvalho de Aguiar 470/500, 05403-000 São Paulo, SP, Brazil \\ ${ }^{2}$ Departamento de Moléstias Infecciosas e Parasitárias, HCFMUSP, Avenida Dr. Enéas Carvalho de Aguiar 255, \\ 05403-000 São Paulo, SP, Brazil \\ ${ }^{3}$ Laboratório de Soroepidemiologia e Imunobiologia, IMTSP-USP, Avenida Dr. Enéas Carvalho de Aguiar 470/500, \\ 05403-000 São Paulo, SP, Brazil \\ ${ }^{4}$ Hospital Emilio Ribas, Avenida Dr. Arnaldo 165, 01246-900 São Paulo, SP, Brazil \\ ${ }^{5}$ Laboratório de Parasitologia, Instituto de Medicina Tropical de São Paulo, Universidade de São Paulo (IMTSP-USP), \\ Avenida Dr. Enéas Carvalho de Aguiar 470/500, 05403-000 São Paulo, SP, Brazil
}

Correspondence should be addressed to Lucia Maria Almeida Braz; lmabraz@usp.br

Received 1 April 2016; Revised 29 June 2016; Accepted 10 July 2016

Academic Editor: José F. Silveira

Copyright (C) 2016 Natalia Souza de Godoy et al. This is an open access article distributed under the Creative Commons Attribution License, which permits unrestricted use, distribution, and reproduction in any medium, provided the original work is properly cited.

\begin{abstract}
The aim of this study was to evaluate whether the molecular (kDNA-PCR) and parasitological diagnosis in peripheral blood (PB) could replace the invasive and painful bone marrow collection (BM) in the diagnosis of visceral leishmaniasis (VL). PB from suspected VL patients was evaluated by parasitological and molecular techniques using as the gold standard (GS) a combination of clinical, epidemiological, and immunochromatographic test (PB-rK39) results and parasitological examination of BM. Based on the GS, 38 samples from 32 patients were grouped: Group 1, 20 samples of VL cases, and Group 2, 18 samples of non-VL cases. In order to evaluate the parasitological and molecular techniques in PB, the samples were examined. From Group 1, PB kDNA-PCR was positive in 20 samples and in 19 of 20 in BM kDNA-PCR examination. However, the parasitological examination of buffy coat was insensitive, being able to detect only 4 cases from Group 1 . All samples from Group 2 were negative. We concluded that, for the diagnosis of visceral leishmaniasis, the parasitological examination of peripheral blood was not useful; however, molecular diagnosis by kDNA-PCR, performed in peripheral blood, could be useful to replace the parasitological examination of bone marrow.
\end{abstract}

\section{Introduction}

Visceral leishmaniasis (VL) has been reported in 88 countries, and $90 \%$ of the world's burden is localized in India, Brazil, and Sudan [1]. In the Americas, VL is known as American Visceral Leishmaniasis (AVL) and its etiologic agent is Leishmania (Leishmania) infantum (L. (L.) chagasi, syn.) [2]. VL is characterized by its chronicity and systemic dissemination capacity [3] and it can be fatal if treatment is not administered. To be correctly diagnosed, VL requires the use of highly sensitive and specific laboratory methods $[4,5]$. In the context of VL, the techniques that are considered gold standards (GS) are the direct microscopic examination of bone marrow (BM) or spleen aspirate samples, with observation of amastigotes on smears (BM-S), and the isolation of promastigotes in culture (BM-C) [6,7]. More recently, the rK39 immunochromatographic test has been included [8]. Although splenic aspirate smears show the highest sensitivity, followed by BM aspirate smears, both exams require invasive and more risky procedures [9-11]. Given the fact that a considerable number of VL patients are children and immunocompromised patients, it would be desirable to adopt less risky and painful procedures provided that similar sensitivity and specificity rates are obtained. Therefore, the 
aim of this study was to evaluate whether parasitological and molecular techniques performed in the BC (buffy coat) or WB (whole blood), from a small volume of peripheral blood $(\mathrm{PB})$, could replace the parasitological examination of the bone marrow (BM), whose collection is invasive and painful, for the diagnosis of visceral leishmaniasis (VL).

Peripheral blood $(\mathrm{PB})$ is a biological material that can be easily collected and used in highly sensitive tests to investigate VL such as Leishmania DNA amplification by PCR performed in whole blood or buffy coat samples [4] and serological detection of anti-Leishmania antibodies [6].

Using samples of whole blood (WB) and buffy coat (BC) from patients with and without VL, parasitological and molecular techniques were evaluated. These samples were used to prepare smears (PB-S) and inoculated in $\mathrm{NNN}$ medium supplemented with $\mathrm{BHI}$ (PB-C). In addition, a kDNA-PCR was performed in $\mathrm{WB}$ and $\mathrm{BC}$ samples (PB kDNA-PCR) as well as in BM samples (BM kDNA-PCR). The performances of these techniques were compared to the GS laboratorial techniques (PB-rK39; BM-S and BM-C) results.

\section{Methods}

2.1. Patients. This research was approved by the Institutional Research Ethics Committee (protocol number 0006/11). After signing the informed consent, participants suspected to have VL coming from different regions of Brazil were attended at the University Hospital (HC-FMUSP) of São Paulo. From 77 samples examined, we selected 38 samples from 32 patients, whose samples from $\mathrm{BM}$ and $\mathrm{PB}$ were matched.

2.2. Laboratorial Gold Standards (GS) and Definition of VL Cases. The laboratorial GS were the parasitological techniques (direct microscopy examination and culture) of BM samples, aside from the immunochromatographic diagnostic test (rK39) in whole blood PB samples (PB-rK39). To be considered a true VL case the participant had to present clinical test, epidemiological test, and at least one positive test among the laboratorial GS.

Thirty-eight samples from 32 patients met the inclusion criteria of the study and were grouped as follows:

Group 1: 20 samples from 18 true VL cases according to the clinical and epidemiological data associated with a positive result in at least one GS laboratorial techniques.

Group 2: 18 samples from 16 non-VL cases presented symptoms initially compatible with VL and had negative results in the GS laboratorial techniques.

2.3. Collection and Processing of Biological Samples. BM aspirates and PB samples $(3.5 \mathrm{~mL})$ were collected in EDTA tubes and submitted to the following diagnostic procedures.

\subsubsection{Parasitological Techniques (Gold Standard When Tested in Bone Marrow)}

Microscopy Examination of the Stained Smear from Bone Marrow (BM-S) and from Peripheral Blood Samples PB (PB$S$ ). Five microliters of $\mathrm{BM}$ and $\mathrm{PB}$ samples (WB and $\mathrm{BC}$ at $1,506 \times \mathrm{g}$ and $20,000 \times \mathrm{g}$, resp.) was used to prepare eight slides smears. From each procedure (BM, WB PB, and BCPB1 at $1,506 \times \mathrm{g}$ and $\mathrm{BCPB} 2$ at $20,000 \times \mathrm{g}) 2$ slides were prepared, which were subsequently stained by Panótico method (Newprov Instant Prov, Paraná, Brazil), a kind of Leishman or Romanowsky dye. They were analyzed by microscopy (1000x magnification), searching for amastigotes, and 200 fields were examined in each smear [12].

Culture of $B M(B M-C)$ and $P B(P B-C)$ in NNN Medium Supplemented with BHI. Forty microliters of BM or the same volume of $B C(1,506 \times g$ and $20,000 \times \mathrm{g})$ from $P B$ samples was inoculated into 2 tubes, containing NNN medium (DIFCO, USA), to which $2 \mathrm{~mL}$ of BHI medium was added (DIFCO, USA). The tubes were incubated in a BOD incubator at $25^{\circ} \mathrm{C}$ and the samples were weekly analyzed for a total of 30 days by means of optical microscopy searching for promastigotes [13].

2.3.2. Molecular Diagnostic Techniques of Peripheral Blood $(P B)$ and Bone Marrow (BM) Polymerase Chain Reaction: $P B$ KDNA-PCR and BM kDNA-PCR. WB and $\mathrm{BC}$ obtained by centrifugation $(1,506 \times \mathrm{g}$ and $20,000 \times \mathrm{g})$ of $\mathrm{PB}$ samples as well as BM samples were submitted to DNA extraction with the aid of the Genomic DNA Extraction Kit (Real Biotech Corporation, Taiwan, China) starting with an initial volume of $300 \mu \mathrm{L}$. DNA samples were identified and stored at $-20^{\circ} \mathrm{C}$. The kDNA primers were designed within a conserved region of Leishmania sp. kDNA minicircles. The forward primer 20 ( $5^{\prime}$-GGGKAGGGGCGTTCTSCGA A-3') and reverse primer $22\left(5^{\prime}\right.$-SSSWCTATWTTACACCAACCCC- $\left.3^{\prime}\right)$ yielded a 120 base-pair amplification product [14].

Amplifications were performed in a total volume of $20 \mu \mathrm{L}$ containing $100 \mathrm{ng}$ of template DNA, $50 \mathrm{mM}$ of $\mathrm{KCl}, 10 \mathrm{mM}$ of Tris-HCl ( $\mathrm{pH} 8.0$ ), $0.2 \mathrm{mM}$ of dNTPs (Fermentas, Thermo Fisher, Ontario, Canada), $1.0 \mathrm{mM}$ of $\mathrm{MgCl}_{2}, 0.4 \mu \mathrm{M}$ of each primer, and 1 unit of Taq DNA Polymerase (Fermentas, Thermo Fisher, Ontario, Canada). In each experiment, two negative controls containing sterile water instead of template DNA were also tested. The positive control was Leishmania infantum DNA extracted from cultures. Each reaction was performed with an initial denaturation step of $94^{\circ} \mathrm{C}$ for 5 minutes, followed by 35 cycles of $94^{\circ} \mathrm{C}$ for 1 minute, $58^{\circ} \mathrm{C}$ for 1 minute, and $72^{\circ} \mathrm{C}$ for 30 seconds, ending with a final extension step of $72^{\circ} \mathrm{C}$ for 5 minutes. Reactions were performed in a minicycler thermocycler (MJ Research Corp/MJ Research, Quebec, Canada) and PCR products were visualized in $2 \%$ ethidium bromide-stained agarose gels by means of a UV transilluminator (Alpha Innotech Corp/Alpha Innotech Multimage, California, USA).

To minimize the risk of contamination, reagents preparation and PCR master mix, DNA extraction, and electrophoresis were performed in three separate areas. To confirm that 
amplification inhibitors were not present, a fragment of the human beta-globin gene was tested in all the samples [15].

2.3.3. Serological Technique. Whole PB samples were tested by the PB-rK39 immunochromatographic kit that uses a recombinant peptide containing 39 amino acid repeats from the kinesin-like gene found in L. chagasi. This test is widely used for VL diagnosis in field studies [11].

2.4. Statistical Analysis. To determine the agreement of tests, the kappa test was used at a 95\% confidence interval and $p$ values $\leq 0.05$ were considered significant. The data were analyzed with the statistical software STATA version 12.0 (Stata Corp LP, College Station, Texas, USA).

\section{Results}

Regarding the GS techniques (Table 1), performed in Group 1 (20 true VL samples), we obtained the following results: 16 samples were positive by BM-S, including 8 also by BM-C; 15 were positive by PB-rK39 (four of which were negative by parasitological examination of $\mathrm{BM}$ ), though there were five samples, from two HIV positive patients, that yielded negative results by PB-rK39 and were positive by parasitological examination of BM. Group 2 was composed of 18 samples from 16 non-VL cases that were negative by all the gold standard techniques (BM-S, BM-C, and PB-rK39). The clinical follow-up of these 16 non-VL cases revealed, in most of them, cutaneous leishmaniasis (1), lupus (2), prostatitis (1), hepatitis (1), urinary tract infection (1), Sjogren syndrome (1), lymphoma (2), gastric ulcers (2), sarcoidosis (1), pellagra (1), pharyngitis (1), hypothyroidism (1), and anemia (1). All DNA samples were amplified by the beta-globin gene demonstrating that there were no amplification inhibitors.

According to Table 1, PB kDNA-PCR, performed in whole blood and buffy coat, was positive in $100 \%$ of Group 1 samples (20/20). PB-S was positive in $20 \%$ of the samples $(4 / 20)$, and PB-C was negative in all samples. The four PB$S$ positive samples were obtained from BCPB1 (one buffy coat sample centrifuged at $1,506 \times \mathrm{g}$ ) and BCPB2 (two buffy coat samples centrifuged at $20,000 \times \mathrm{g}$ ) and one in BCPB1 and BCPB2 (buffy coat sample tested after centrifugation at $1,506 \times \mathrm{g}$ and $20,000 \times \mathrm{g}$ ). It is noteworthy that none of these four samples was parasitologically positive when the analysis was performed directly in the corresponding whole blood samples.

Analyzing the PB kDNA-PCR and BM kDNA-PCR they presented a specificity of $100 \%$ and a sensitivity of $100 \%$ and $95 \%$, respectively (Table 2).

A good concordance, with a kappa index of 0,79 and 0,74 , was obtained $(p<0.001)$, when PB kDNA-PCR was compared with the gold standard techniques: the BM-S and the rK39, respectively (Table 3). Comparison between PB kDNA-PCR and BM kDNA-PCR results showed an almost perfect correlation $(0.94, p<0.001)$ (Table 3$)$.

\section{Discussion}

In this study $\mathrm{PB}$ samples were tested as a substitute of invasive and painful procedures to obtain BM samples for the diagnosis of VL. Abeijon and Campos-Neto [16] investigated a potential noninvasive urine-based antigen detection assay to diagnose active VL. One of the advantages of PB samples to investigate $\mathrm{VL}$ is that the same biological material can be concomitantly analyzed by parasitological, serological, and molecular techniques unlike urine samples. The main symptoms of VL patients in our study were hepatosplenomegaly, febrile, and pancytopenia as presented for all the patients in Group 1 (true VL cases, Table 1). They were all Brazilians and came from VL endemic regions $[2,3]$.

Regarding the parasitological investigation in peripheral blood, buffy coat PB-S detected $20 \%$ of positive samples, while none of the samples were positive when whole blood samples were examined, as expected, because parasites are concentrated in buffy coat facilitating the visualization of amastigotes within leucocytes [4]. Nevertheless, 20\% detection is very low when compared to BM-S, one of the gold standards ( $80 \%$ of positivity). Similarly, PB-C did not find any positive sample while BM-C detected $40 \%$. Sixteen out of 20 samples were negative by $\mathrm{PB}-\mathrm{S}$, and this low sensitivity can be explained by the fact that there are more parasites in the bone marrow and spleen samples $[6,7]$, justifying why they are the gold standard of VL laboratorial investigation. In the present study, cultures were not a sensitive method compared to other techniques, even in the case of BM, as this exam was able to confirm only $40 \%$ of truly positive samples. Aside from being very prone to contamination, cultures are time consuming requiring four weeks to release a final result, what has already been acknowledged by other authors [17] and was corroborated in this research.

According to the ideal molecular target for detecting Leishmania, kDNA was chosen due to the large presence of minicircles in the cells of the parasite, about $10^{4}$ copies per cell [17]. Moreover, the proven viability of parasites in PB, which is a biological material, obtained more easily than the $\mathrm{BM}$ leads us to search for kDNA in the PB. Nevertheless, differently of parasitological investigation in $\mathrm{PB}$, molecular investigation (kDNA) in $\mathrm{PB}$ did not present differences between buffy coat (BCPB: $1506 \times \mathrm{g}$ and BCPB: $20000 \times \mathrm{g}$ ) and whole blood. All samples 20/20 were positive. According to Srivastava et al. [4] PCR analysis of the whole blood or its buffy coat preparation may prove a useful screening test. A sensitivity of $100 \%$ was obtained by PB kDNA-PCR when compared to the gold standard techniques (BM-S, BM-C, and PB-rK39). By comparing molecular and parasitological techniques, Ozerdem et al. [18] obtained better results with kDNA-PCR (29/50 or 58\%) in comparison with microscopic examination (10/50 or 20\%) of Giemsa-stained smears from blood samples of suspected VL patients. In our study, PB kDNA-PCR showed a good concordance with the rapid immunochromatographic test (PB-rK39) $(0.74, p<0.001)$, which is a rapid and highly sensitive technique, so that it has been used as a reference test. Disch et al. [9] and Andresen et al. [19] obtained sensitivities of 91\% (48/53) and 92.5\% (37/40) when kDNA-PCR was used to test whole blood from 


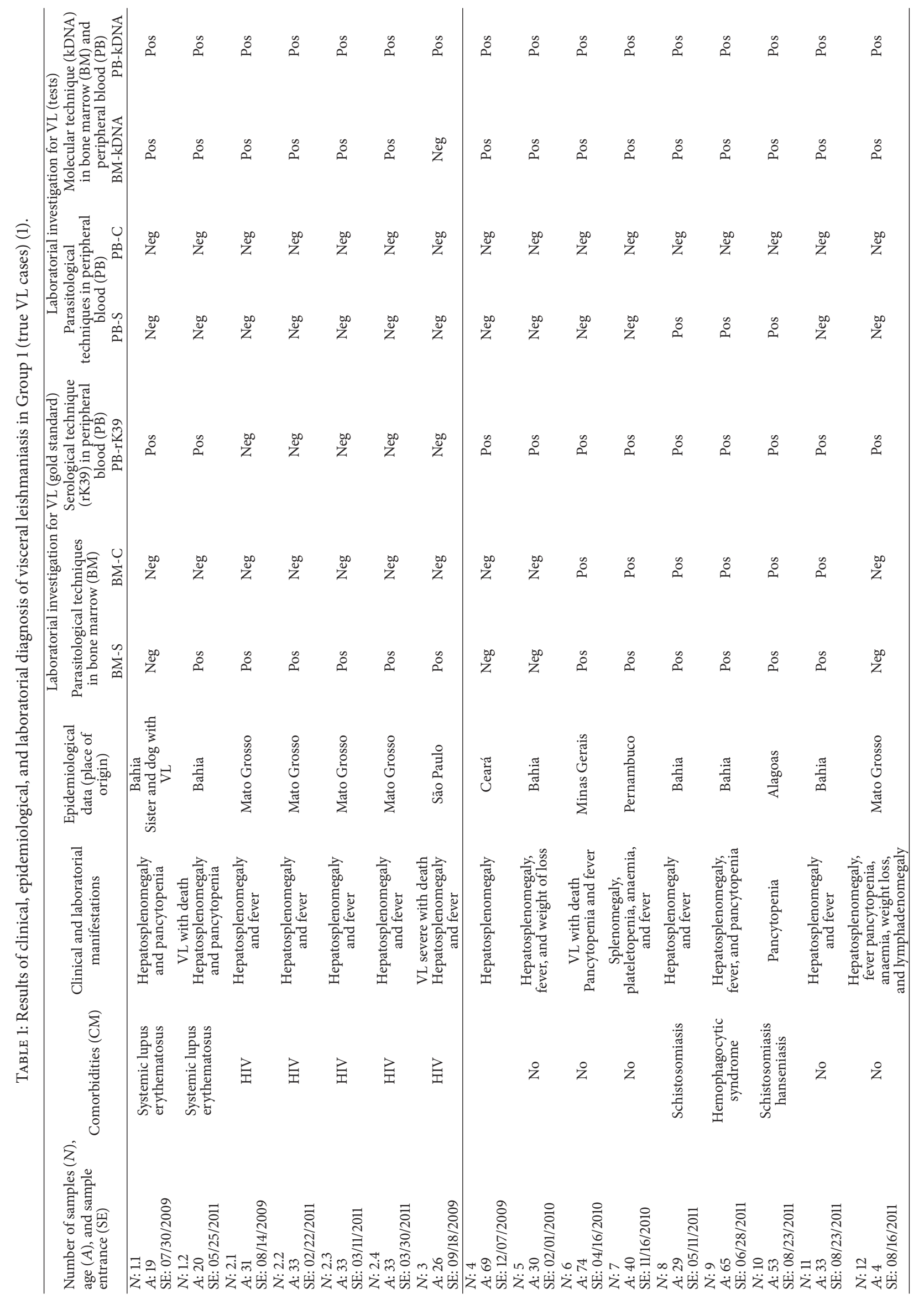




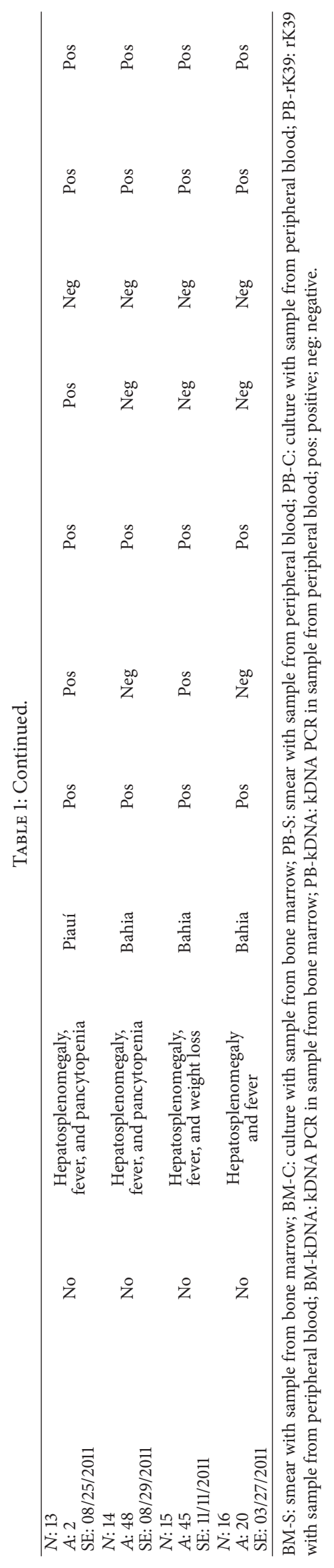


TABLE 2: Sensitivity, specificity, predictive positive value, predictive negative value, probability of false positive, probability of false negative of PB kDNA-PCR and BM kDNA-PCR.

\begin{tabular}{lccccccc}
\hline Test & Sensitivity (\%) & Specificity (\%) & PPV (\%) & PNV (\%) & PFP (\%) & PFN (\%) & Efficiency (\%) (CI 95\%) \\
\hline PB kDNA-PCR & 100 & 100 & 100 & 100 & 0 & 0 & $100(90.7-100)$ \\
BM kDNA-PCR & 95.0 & 100 & 100 & 95.2 & 0 & 4.8 & $97.3(86.2-99.9)$ \\
\hline
\end{tabular}

BM kDNA-PCR: kDNA PCR in sample from bone marrow; PB kDNA-PCR: kDNA PCR in sample from peripheral blood; PPV: predictive positive value; PNV: predictive negative value; PFP: probability of false positive; PFN: probability of false negative; CI 95\%: 95\% confidence intervals.

TABLE 3: Comparative analysis between the results of PB kDNA-PCR and results of BM kDNA-PCR, BM-S, BM-C, and PB-rk39.

\begin{tabular}{|c|c|c|c|c|c|c|}
\hline \multirow{2}{*}{ Test } & \multicolumn{3}{|c|}{ PB kDNA-PCR } & \multirow{2}{*}{ Kappa (CI 95\%) } & \multirow{2}{*}{$p$ value } & \multirow{2}{*}{ Efficiency (\%) (CI 95\%) } \\
\hline & Positive & Negative & Total & & & \\
\hline BM kDNA-PCR & & & & 0.947 & $<0.001$ & $97.4(86.2-99.9)$ \\
\hline Positive & 19 & 0 & 19 & \multirow{3}{*}{ Almost perfect correlation } & & \\
\hline Negative & 1 & 18 & 19 & & & \\
\hline Total & 20 & 18 & 38 & & & \\
\hline BM-S & & & & 0.791 & $<0.001$ & 89.5 (75.2-97.1) \\
\hline Positive & 16 & 0 & 16 & $(0.602-0.980)$ & & \\
\hline Negative & 4 & 18 & 22 & \multirow{2}{*}{ Good concordance } & & \\
\hline Total & 20 & 18 & 38 & & & \\
\hline BM-C & & & & 0.387 & 0.001 & $68.4(51.3-82.5)$ \\
\hline Positive & 8 & 0 & 8 & $(0.160-0.614)$ & & \\
\hline Negative & 12 & 18 & 30 & \multirow{2}{*}{ Low concordance } & & \\
\hline Total & 20 & 18 & 38 & & & \\
\hline PB-rK39 & & & & 0.740 & $<0.001$ & $86.4(71.9-95.6)$ \\
\hline Positive & 15 & 0 & 15 & $(0.534-0.945)$ & & \\
\hline Negative & 5 & 18 & 23 & \multirow{2}{*}{ Good concordance } & & \\
\hline Total & 20 & 18 & 38 & & & \\
\hline
\end{tabular}

BM kDNA-PCR: kDNA PCR in sample from bone marrow; PB kDNA-PCR: kDNA PCR in sample from peripheral blood; PB-rk39: rK39 with sample from peripheral blood; CI 95\%: 95\% confidence intervals.

PB samples of patients with VL, confirmed by clinical and microscopic examination of BM or lymph node samples. Fraga et al. [20] evaluated the effectiveness of a kDNA-PCR in PB and found a very good sensitivity (43/45; 95.6\%), which was higher than that found in BM samples: kDNA (41/45; 91.1\%); microscopic examination of smear (36/45; $80 \%)$; and culture $(12 / 45 ; 26.7 \%)$. Antinori et al. [21] used PCR and obtained a sensitivity of $98.5 \%(64 / 65)$ and $95.7 \%$ (45/47) in $\mathrm{PB}$ and $\mathrm{BM}$, respectively, once again confirming the better sensitivity of PB-PCR (whole blood) in comparison with BMPCR. In contrast, Cruz et al. [10] found a higher positivity of Ln-PCR in BM (24/24) than in whole blood of PB (Ln-PCR) $(19 / 24)$.

By means of immunochromatography test, due to its sensitivity and rapidity, the PB-rK39 can screen suspected cases of VL, especially in immunocompetent patients. Although it is not our main objective, it is necessary to reinforce that the presence of VL antibodies, associated with clinical and epidemiological data, can assist in prompt medical decisions, but it cannot differentiate past from active infection. In order to diagnose an active VL infection in $\mathrm{PB}$ samples, from immunocompetent or immunodeficient patients, kDNAPCR is the most appropriate. Unlike the BM, it also presents the advantage of being an easy to take biological sample. Nonetheless, parasitological examination of the peripheral blood (PB-S and PB-C) cannot substitute the parasitological examination of bone marrow (BM-S and BM-C).

In conclusion, kDNA-PCR performed in small volumes of $\mathrm{PB}$, in either whole blood or buffy coat, showed a good agreement with VL gold standard tests. Therefore peripheral blood could be useful to replace the invasive and painful procedures to obtain bone marrow samples for the diagnosis of visceral leishmaniasis.

\section{Ethical Approval}

Ethics Committee of HCFMUSP-BRASIL (given number 0006/11 CAPPesq) approved this study.

\section{Competing Interests}

The authors declare that they have no competing interests.

\section{Acknowledgments}

The authors thank Professor Dr. Thelma S. Okay for her help in correction of the English language and Edite H. Y. Kanashiro, M.S., for providing help in the parasitological examination, the "Laboratório de Micologia (LIM-53)" and 
the "Laboratório de Bacteriologia (LIM 54)". The research project was conducted fully sponsored by FAPESP (Fundação de Amparo à Pesquisa do Estado de São Paulo) with Grant no. 2010-50304-8 under the supervision of Lucia Maria Almeida Braz.

\section{References}

[1] S. K. Bhattacharya, D. Sur, P. K. Sinha, and J. Karbwang, "Elimination of leishmaniasis (kala-azar) from the Indian subcontinent is technically feasible \&operationally achievable," Indian Journal of Medical Research, vol. 123, no. 3, pp. 195-196, 2006.

[2] G. Michel, C. Pomares, B. Ferrua, and P. Marty, "Importance of worldwide asymptomatic carriers of Leishmania infantum ( $L$. chagasi) in human," Acta Tropica, vol. 119, no. 2-3, pp. 69-75, 2011.

[3] A. N. S. Maia-Elkhoury, W. A. Alves, M. L. De Sousa-Gomes, J. M. De Sena, and E. A. Luna, "Visceral leishmaniasis in Brazil: trends and challenges," Cadernos de Saude Publica, vol. 24, no. 12, pp. 2941-2947, 2008.

[4] P. Srivastava, A. Dayama, S. Mehtotra, and S. Sundar, "Diagnosisof visceral leishmaniasis," Transactions of the Royal Society of Tropical Medicine and Hygiene, vol. 105, no. 1, pp. 1-6, 2011.

[5] F. Chappuis, S. Sundar, A. Hailu et al., "Visceral leishmaniasis: what are the needs for diagnosis, treatment and control?" Nature Reviews Microbiology, vol. 5, no. 11, pp. 873-882, 2007.

[6] T. S. M. de Assis, R. B. Caligiorne, G. A. S. Romero, and A. Rabello, "Detection of Leishmania kDNA in human serum samples for the diagnosis of visceral leishmaniasis," Transactions of the Royal Society of Tropical Medicine and Hygiene, vol. 103, no. 12, pp. 1269-1272, 2009.

[7] M. N. Rocha, C. Margonari, I. M. Presot, and R. P. Soares, "Evaluation of polymerase chain reaction protocols for cultured Leishmania spp. typing," Diagnostic Microbiology and Infectious Disease, vol. 68, no. 4, pp. 401-409, 2010.

[8] T. S. M. Assis, A. S. C. Braga, M. J. Pedras et al., "Validation of the rapid immunochromatographic test IT-LEISH ${ }^{5}$ for the diagnosis of human visceral leishmaniasis," Epidemiologia $e$ Serviços de Saúde, vol. 17, no. 2, pp. 107-116, 2008.

[9] J. Disch, F. C. Maciel, M. C. de Oliveira, M. Orsini, and A. Rabello, "Detection of circulating Leishmania chagasi DNA for the non-invasive diagnosis of human infection," Transactions of the Royal Society of Tropical Medicine and Hygiene, vol. 97, no. 4, pp. 391-395, 2003.

[10] I. Cruz, C. Chicharro, J. Nieto et al., "Comparison of new diagnostic tools for management of pediatric mediterranean visceral leishmaniasis," Journal of Clinical Microbiology, vol. 44, no. 7, pp. 2343-2347, 2006.

[11] K. Ritmeijer, Y. Melaku, M. Mueller, S. Kipngetich, C. O’Keeffe, and R. N. Davidson, "Evaluation of a new recombinant K39 rapid diagnostic test for Sudanese visceral leishmaniasis," American Journal of Tropical Medicine and Hygiene, vol. 74, no. 1, pp. 76-80, 2006.

[12] São Paulo, "Manual de vigilância e controle da leishmaniose visceral americana do estado de São Paulo," in Secretaria de Estado da Saúde de São Paulo, Superintendência de Controle de Endemias/SUCEN, e Coordenadoria de Controle de Doenças/CCD, Vera Lucia Fonseca de Camargo-Neves, 2006.

[13] A. K. Boggild, C. Miranda-Verastegui, D. Espinosa et al., "Optimization of microculture and evaluation of miniculture for the isolation of Leishmania parasites from cutaneous lesions in Peru," The American Journal of Tropical Medicine and Hygiene, vol. 79, no. 6, pp. 847-852, 2008.

[14] A. C. Nicodemo, V. S. Amato, F. F. Tuon, R. M. Souza, T. S. Okay, and L. M. Braz, "Usefulness of kDNA PCR in the diagnosis of visceral leishmaniasis reactivation in co-infected patients," Revista do Instituto de Medicina Tropical de São Paulo, vol. 55, no. 6, pp. 429-431, 2013.

[15] S. Nakajima-Iijima, H. Hamada, P. Reddy, and T. Kakunaga, "Molecular structure of the human cytoplasmic $\beta$-actin gene: Interspecies homology of sequences in the introns," Proceedings of the National Academy of Sciences of the United States of America, vol. 82, no. 18, pp. 6133-6137, 1985.

[16] C. Abeijon and A. Campos-Neto, "Potential non-invasive urinebased antigen (Protein) detection assay to diagnose active visceral leishmaniasis," PLoS Neglected Tropical Diseases, vol. 7, no. 5, Article ID e2161, 2013.

[17] G. Schönian, A. Nasereddin, N. Dinse et al., "PCR diagnosis and characterization of Leishmania in local and imported clinical samples," Diagnostic Microbiology and Infectious Disease, vol. 47, no. 1, pp. 349-358, 2003.

[18] D. Ozerdem, F. Eroglu, A. Genc, M. Demirkazik, and I. S. Koltas, "Comparison of microscopic examination, rK39, and PCR for visceral leishmaniasis diagnosis in Turkey," Parasitology Research, vol. 106, no. 1, pp. 197-200, 2009.

[19] K. Andresen, S. Gasim, A. M. Elhassan et al., "Diagnosis of visceral leishmaniasis by the polymerase chain reaction using blood, bone marrow and lymph node samples from patients from the Sudan," Tropical Medicine and International Health, vol. 2, no. 5, pp. 440-444, 1997.

[20] T. L. Fraga, Y. M. Brustoloni, R. B. Lima et al., "Polymerase chain reaction of peripheral blood as a tool for the diagnosis of visceral leishmaniasis in children," Memorias do Instituto Oswaldo Cruz, vol. 105, no. 3, pp. 310-313, 2010.

[21] S. Antinori, S. Calattini, E. Longhi et al., "Clinical use of polymerase chain reaction performed on peripheral blood and bone marrow samples for the diagnosis and monitoring of visceral leishmaniasis in HIV-infected and HIV-uninfected patients: a single-center, 8-year experience in italy and review of the literature," Clinical Infectious Diseases, vol. 44, no. 12, pp. 1602-1610, 2007. 

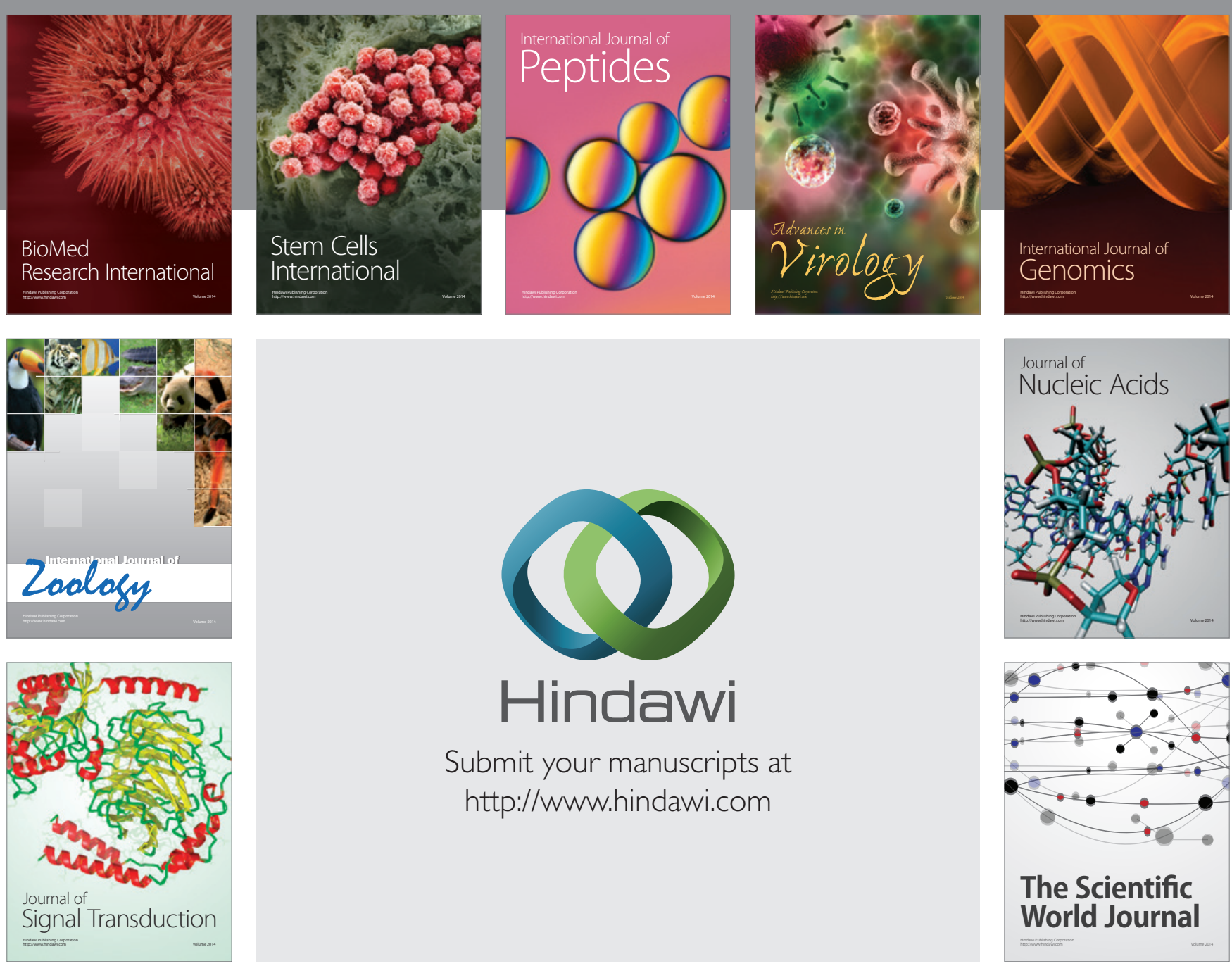

Submit your manuscripts at

http://www.hindawi.com
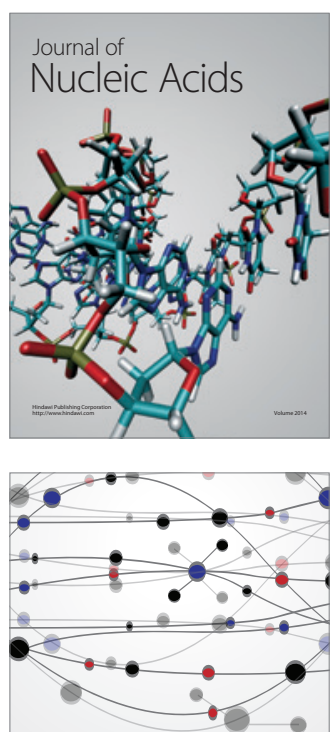

The Scientific World Journal
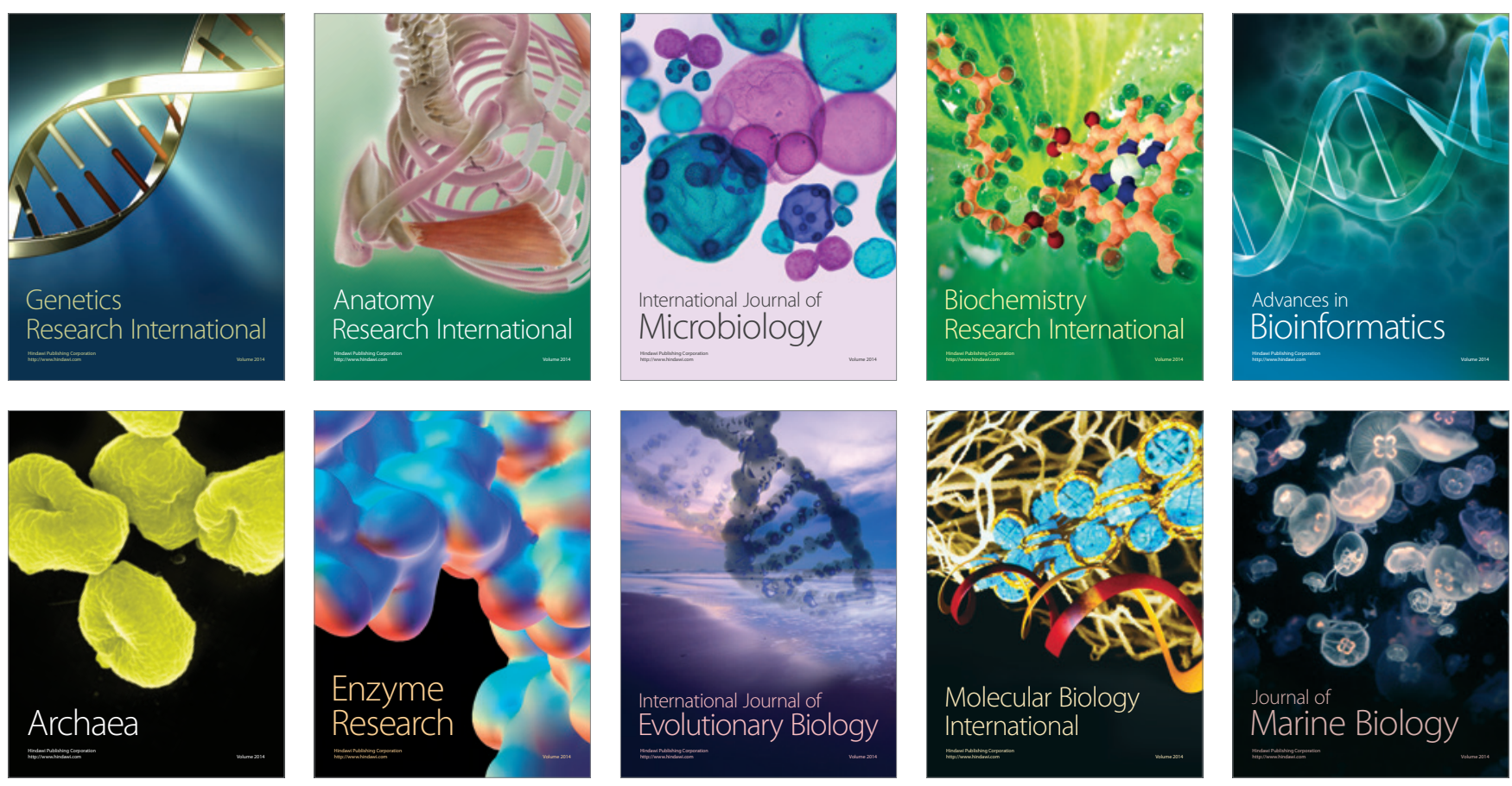